\title{
Performance Analysis of a Hardware Implemented Complex Signal KURTOSIS RADIO-FREQUENCY INTERFERENCE DETECTOR
}

NASA

Adam J. Schoenwald ${ }^{1}$, Adam.Schoenwald@nasa.gov

Damon C. Bradley ${ }^{1}$, Priscilla N. Mohammed ${ }^{1,2}$, Jeffrey R. Piepmeier ${ }^{1}$, Mark Wong

(1) NASA Goddard Space Flight Center, Greenbelt, MD (2) Goddard Earth Sciences Technology and Research, Morgan State University

\section{Motivation}

RFI compromises quality of science products.

\section{Spectrum is becoming} crowded and shared.

Hardware capabilities allow for digital radiometry.

Need more sensitive detectors for wide-band interference.

\section{Complex Signal Kurtosis}

Given a complex baseband signal $z(n)=I(n)+$ $j Q(n)$, moments $\alpha_{\ell, m}$ of $z(n)$ are defined as

$$
\alpha_{\ell, m}=\mathbb{E}\left[(z-\mathbb{E}[z])^{\ell}(z-\mathbb{E}[z])^{* m}\right], \ell, m \in \mathbb{R} \geq 0
$$

With $\sigma^{2}=\alpha_{1,1}$, Standardized moments $\varrho_{\ell, m}$ can then be found as

$$
\varrho_{\ell, m}=\frac{\alpha_{\ell, m}}{\sigma^{\ell+m}}
$$

Leading to the CSK (Complex Signal Kurtosis) rfi test statistic used $[1,2]$

$$
C_{K}=\frac{\varrho_{2 ; 2}-2-\left|\varrho_{2 ; 0}\right|^{2}}{1+\frac{1}{2}\left|\varrho_{2 ; 0}\right|^{2}}
$$

\section{Real Signal Kurtosis}

Given a complex baseband signal $z(n)=I(n)+$ $j Q(n)$, the fourth standardized moment is computed independently for both the real and imaginary vectors, $I$ and $Q$ as was used in SMAP[3].

$$
\mathrm{RSK}_{\mathrm{I}}=\frac{\mathbb{E}\left[\left(\mathrm{I}-\mathbb{E}[[])^{4}\right]\right.}{(\mathbb{E}[(\mathrm{I}-\mathbb{E}[[]])])^{2}}-3, \mathrm{RSK}_{\mathrm{Q}}=\frac{\mathbb{E}\left[(\mathrm{Q}-\mathbb{E}[\mathrm{Q}])^{4}\right]}{(\mathbb{E}[(\mathrm{Q}-\mathbb{E}[\mathrm{Q}])])^{2}}-3
$$

The test statistic, RSK (Real Signal Kurtosis), is then defined as

$$
\mathrm{RSK}=\frac{\left|\mathrm{RSK}_{\mathrm{I}}\right|+\left|R S K_{Q}\right|}{2}
$$
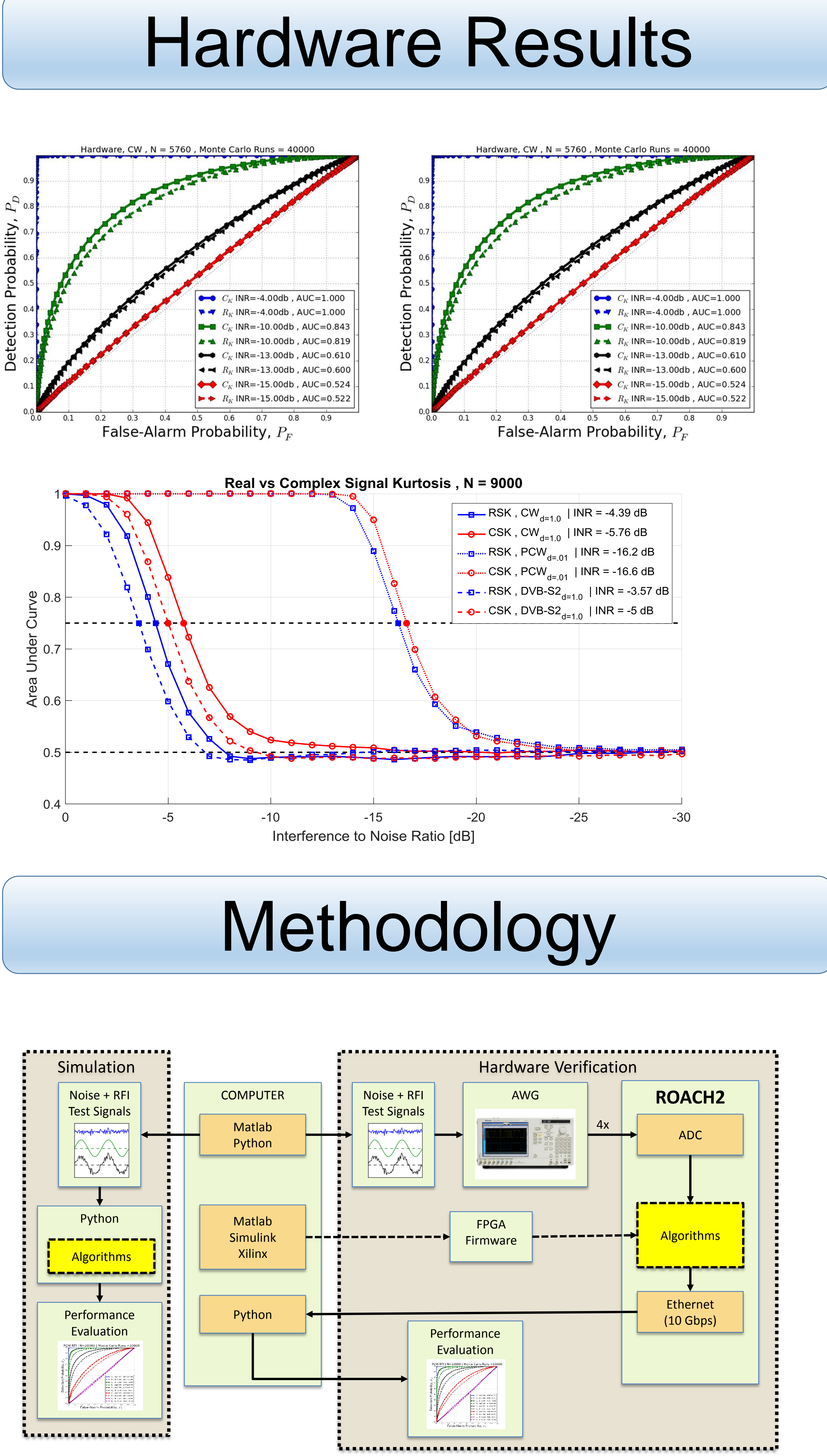

\section{Moment Calculation}

Using the nomenclature for raw moments of the rth power, $m I^{r}=\mathbb{E}\left[I^{p}\right], m Q^{r}=\mathbb{E}\left[Q^{p}\right]$, full band moments produced to compute kurtosis include.

$$
\left\{m I^{r}, m Q^{r}\right\}, \quad r \in\{1,2,3,4\}
$$

Additionally the following cross complex moments are generated.

$$
\{m I Q, m I Q Q, m I I Q, m I I Q Q\}
$$

In the case of sub-banding, all 12 moments for each polarization are produced for every sub-band.

\section{Simulation Results}
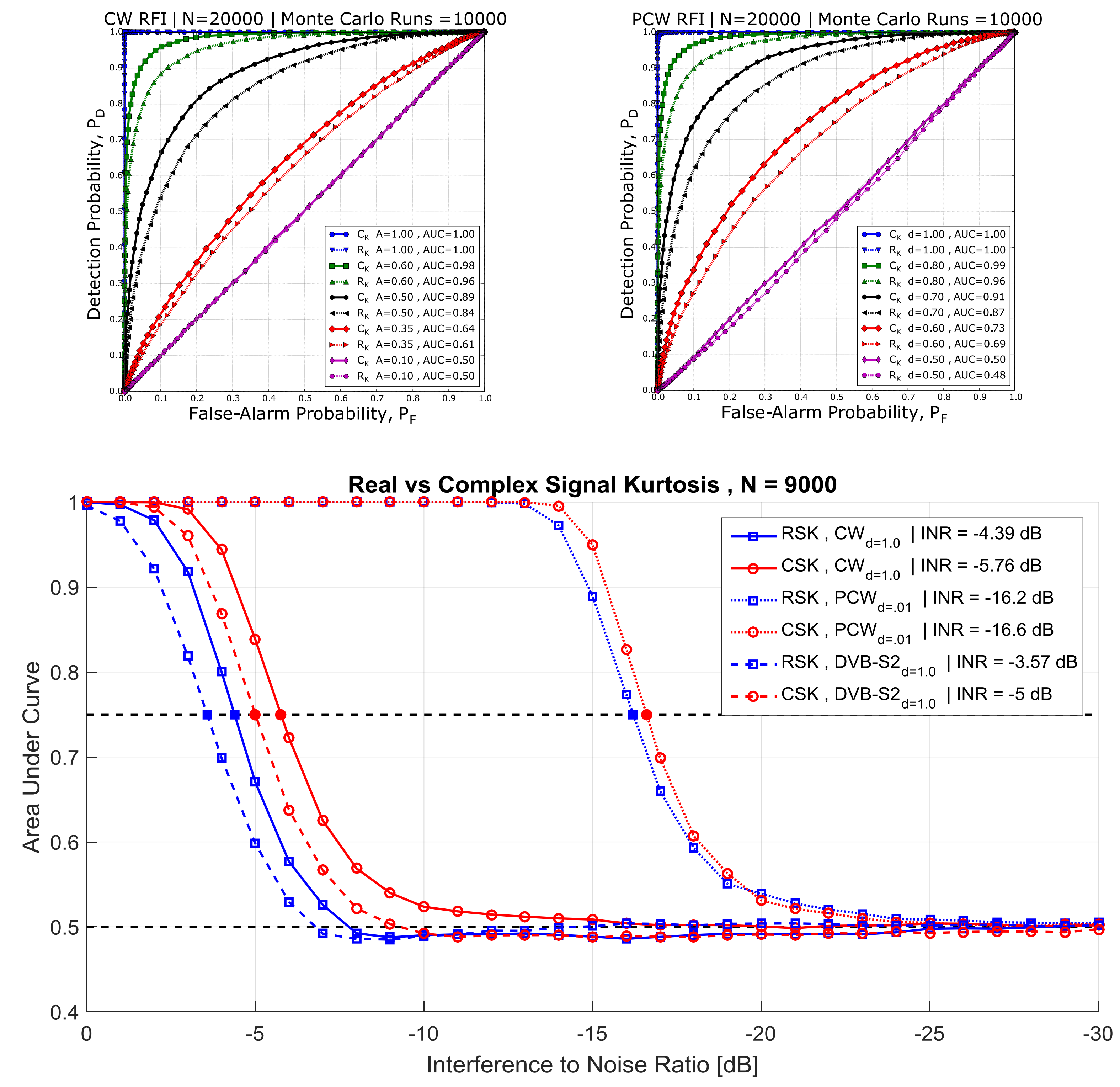

\section{Conclusions}

CSK (Complex Signal Kurtosis) provides a better detection rate than real signal kurtosis.

Interference becomes detectable at an INR (Interference to Noise Ratio) of $2 \mathrm{~dB}$ lower than what can be detected using RSK (Real Signal Kurtosis).

\section{References}

D. C. Bradley, A. J. Schoenwald, M. Wong, P. N. Mohammed and J. R. Piepmeier, "Wideband digital signal processing test-BED for radiometric RF mitigation," Geoscience and Remote Sensing Symposium (IGARSS), 2015

2. E. Ollila, J. Eriksson and V. Koivunen, "Complex Elliptically Symmetric Random Variables-Generation, Characterization, and Circularity Tests," in 011 .

J. Piepmeier, J. Johnson, P. Mohammed, D. Bradley, C. Ruf, M. Aksoy, R. Garcia, D. Hudson, L. Miles, and M. Wong, "Radio-frequency interference mitigation for the soil moisture active passive microwave radiometer," IEEE Transactions on Geoscience and Remote Sensing, vol. 52, no. 1, pp. 76 Acknowledgments

The research team would like to thank the NASA Earth Science Technology Office NNH13ZDA001NACT program for funding this research. 